\title{
IMPUTACIÓN OBJETIVA DE LOS DAÑOS POR INCUMPLIMIENTO CONTRACTUAL
}

\section{OBJECTIVE IMPUTATION OF THE DAMAGES BY BREACH OF CONTRACT}

\author{
FABIÁN GONZÁLEZ CAZORLA*
}

\begin{abstract}
RESUMEN: En estas líneas se comenta una reciente sentencia de la Corte Suprema de fecha 24 de enero de 2019, que se pronuncia sobre la imputación objetiva del daño moral ante el incumplimiento contractual. Se concluye que el máximo tribunal efectúa un correcto análisis causal para determinar la extensión de los daños reparables, al recurrir, precisamente, a los criterios normativos de imputación y la magnitud de la lesión sufrida por el acreedor.
\end{abstract}

Palabras clave: incumplimiento, causalidad, imputación objetiva, daño moral

ABSTRACT: In this work it's comment a recent sentence of the Supreme Court of January 24th, 2019, which refer on the objective imputation of moral damage from breach of contract. It is concluded that the Court does a correct causal analysis to determine the extent of the compensable damage, resorting, to the normative standards of imputation and the severity of the injury suffered by the creditor.

Key words: breach of contract, causality, objective imputation, non-pecuniary loss

\section{INTRODUCCIÓN}

El objeto de este comentario de sentencia consiste en demostrar particularmente dos cosas. La primera, se refiere a que el estado actual de la doctrina y la jurisprudencia no responden de una forma completa y suficiente a la pregunta acerca de los daños indemnizables por incumplimiento contractual, especialmente del daño moral o extrapatrimonial. La segunda, alude a que algunos fallos de la Corte Suprema -pero específicamente el que se analiza en este trabajo- intentan entregar herramientas que subsanen la deuda pendiente sobre la delimitación del daño moral derivado de la infracción de un contrato, esto es, mediante criterios de previsibilidad y causalidad material y normativa que atribuyan el efecto dañoso al incumplimiento del deudor.

Para cumplir estos objetivos conviene mostrar el estado de cosas existente sobre la materia, de manera que nos permita situar la discusión que suscita la resarcibilidad de los daños y los criterios habitualmente utilizados por los tribunales a la hora de fijar los límites de la pretensión indemnizatoria del acreedor. En consecuencia, el trabajo se articulará considerando primeramente el estado de la discusión; luego se hará referencia a la sentencia y al comentario de la misma, para finalizar con algunas conclusiones.

\footnotetext{
* Abogado. Magíster en Derecho Privado por la Universidad de Chile. Profesor de Derecho Civil en la Facultad de Derecho de la Universidad Andrés Bello. Dirección postal: Avenida Bellavista No 0121, Providencia, Santiago, Dirección electrónica: fabian.gonzalez.c@unab.cl
} 


\section{ESTADO DE COSAS: EL DAÑO MORAL EN EL DERECHO CONTRACTUAL}

La procedencia del daño moral por incumplimiento contractual es algo que hoy no se discute. Si bien durante la segunda mitad del siglo XX hubo posiciones encontradas respecto de la reparación de este perjuicio (algunos negándolo ${ }^{1}$ y otros aceptando su procedencia ${ }^{2}$ ), con las sentencias de la Corte Suprema del año $1994^{3}$ y $2001^{4}$ se sentaron las bases de un nuevo paradigma que cambió el derecho chileno de contratos, extendiendo el abanico de protección del acreedor ${ }^{5}$.

No obstante, ello, aún existen problemas en torno a este perjuicio, pero estos se relacionan a otros puntos: aquellos referidos a los límites del daño indemnizable, la previsibilidad y la relación de causalidad entre el incumplimiento y el daño ${ }^{6}$. Tres cuestiones que, incluso los fallos más relevantes, dejaron pendiente en esta materia.

Por este motivo, el escenario en el que nos encontramos plantea determinar aquello que constituye un daño moral indemnizable a la luz de los presupuestos de la responsabilidad civil, junto con los criterios normativos de imputación mediante los cuales se fijen los límites de la protección contractual, esto es, qué intereses del acreedor están protegidos por el contrato cuya lesión - por medio del incumplimiento del deudor-son hábiles para causar un daño extrapatrimonial.

Esta pregunta atañe a uno de los presupuestos de la responsabilidad contractual que no ha sido estudiado con la debida profundidad: la relación de causalidad ${ }^{7}$. Lógicamente, existen aproximaciones dogmáticas ${ }^{8}$ que entregan herramientas útiles para afrontar el

\footnotetext{
1 En la posición que negaba la procedencia de la indemnización del daño moral por incumplimiento contractual Butrón señala que conforme al artículo 1556 del Código Civil "Habría lugar a la indemnización, por consiguiente, sólo en este caso, es decir, habiendo daño emergente o lucro cesante, lo que no podría establecerse, por ejemplo, en caso de no restitución de retratos de familia sin valor apreciable en dinero; pero sí de un gran valor de afección”. Véase BUTRÓN (2009) p. 146. En el mismo sentido ALESSANDRI (1943) p. 547. También BARROS (1932) p. 89. Al respecto el autor señala lo siguiente "El tercer elemento constitutivo de la responsabilidad civil del deudor es la existencia de perjuicios efectivos sufridos por el acreedor en su patrimonio, con motivo del incumplimiento de la obligación. Perjuicios, en general, son los daños que el acreedor ha sufrido en su patrimonio y que han disminuido la hacienda que tenía, o la que legítimamente esperaba tener, si hubiera cumplido la obligación, en la forma específica en que fue contraída; y decimos en su patrimonio, porque no son indemnizables, ni pueden estimarse en dinero, que es la manera de pagar los perjuicios, los daños puramente morales".

2 Se trata de Fueyo, quien alude a la aceptación del daño moral contractual y hace referencia a la evolución de la doctrina francesa que ya lo había acogido para algunos casos (véase FUEYO (1965) p. 72 y FUEYO (1972), p. 26).

3 Véase RAFART CON BANCO DE CHILE (1994); DE LA MAZA (2018a) p. 275.

4 RUIZ CON LABORATORIO BIOLÓGICO S.A. Y OTROS (2001). El considerando 17o señala lo siguiente: "El citado artículo 1556 del Código Civil, como ya quedó establecido precedentemente, no limita la reparación en materia contractual al daño emergente y lucro cesante, desde luego no excluye al daño moral".

5 Para una visión panorámica de la evolución de nuestra jurisprudencia en esta materia véase De la Maza (2018a)

6 GonZÁlez (2019) p. 169

7 Por ejemplo, el profesor Carlos Pizarro indica respecto de la previsibilidad en materia contractual que "La regla del artículo $1558 \mathrm{CC}$. ha tenido muy poca atención de la doctrina nacional, siendo también escasa su aplicación jurisprudencial como límite a la indemnización de los daños contractuales. Incluso no pocos son los que han abogado por su eliminación, entiendo que constituye una regla inútil e innecesaria”. Véase PIZARRO (2013) p. 78.

8 Barrientos (2007) pp. 7-22; Corral (2009) pp. 139-159; Corral (2010) pp. 151 y ss. De la MaZA (2018a) pp. 275-309; DE LA MAZA (2018b) pp. 635-654.
} 
gran desafío respecto de la determinación del daño indemnizable y su extensión, pero no hay claridad sobre cómo proceder más que la tradicional regla de la previsibilidad del artículo 1558 del Código Civil ${ }^{9}$, cuya aplicación por los tribunales suele articularse sobre una noción subjetiva en la persona del deudor ${ }^{10}$, similar a la contemplation rule del Common $l a w^{11}$, aunque, en cierta forma, dicho juicio ha ido cambiando paulatinamente, dando espacio a uno de carácter objetivo ${ }^{12}$.

Sin ir más lejos, en su intento por delimitar los daños contractuales, la Corte Suprema parece haber ido moldeando este criterio a través de una sentencia del año 2012 que excluía la indemnización del daño moral por no resultar previsible para el deudor, por cuanto no estaba dentro de los riesgos asumidos por éste. En dicha sentencia se lee:

"Que no se discute ya en doctrina que es posible que el incumplimiento contractual pueda dar lugar a una indemnización por daño moral, si bien la respuesta acerca de la procedencia de la misma no puede formularse en términos absolutos, pues la naturaleza y el tipo específico de contrato serán relevantes a la hora de determinar si el menoscabo moral que se pretende derivado del incumplimiento de una obligación determinada resultaba o no previsible para los contratantes, es decir, si el acreedor de la indemnización que se demanda tomó a su cargo el riesgo de lesionar intereses extrapatrimoniales de su contraparte"13.

La Corte de una manera elocuente presenta el problema de la delimitación de los daños a través de una regla objetiva de previsibilidad, fundada en los riesgos asumidos por el deudor. Sin embargo, esta postura no ha sido uniforme, porque los tribunales consideran diversos elementos para determinar el daño indemnizable y, generalmente ha sido la naturaleza de la prestación un elemento preponderante a la hora de determinarlo ${ }^{14}$.

\section{EL CASO Y LAS SENTENCIAS DE PRIMER Y SEGUNDO GRADO}

\section{HECHOS RELEVANTES}

De acuerdo a los antecedentes esgrimidos en el proceso, los hechos se pueden sintetizar de la forma que sigue. Doña Natalia Parra Vásquez concurrió el día 30 de abril de 2012 a un centro gineco obstétrico con el objeto de practicarse una ecografía y mostrarle a

\footnotetext{
9 CORRAL (2009) p. 146.;

10 Ejemplo, ZORIN S.A CON COMPAÑÍA SIDERÚRGICA HUACHIPATO (2012), considerando 18: "La frustración de un negocio de esta naturaleza parecía perfectamente previsible, al tiempo de celebrar el contrato, para los efectos del artículo 1558 del Código Civil, pues, era natural que la empresa compradora quisiera revender el material de chatarra que estaba adquiriendo".

11 BURROWs (2005) p. 94 "There is a single or kind of loss is not too remote a consequence of a breach of contract if, at the time of contracting (and on the assumption that the parties actually foresaw the breach in question) it was within their reasonable contemplation as a not unlikely result of that breach"

12 En un tono crítico CÁRDENAS y REVECo (2018) p. 408, señalan que "Por una parte, se ha criticado la inseguridad de la regla de la previsibilidad, en el sentido de que no explicaría satisfactoriamente porqué en determinados casos un daño es indemnizable y en otros no; y, por otro lado, la poca importancia que se presta al contrato como instrumento de distribución de los riesgos contractuales".

13 BAZAN CON SOCIEDAD COMERCIAL CONCESIONARIA SUbTERRA S.A. (2012), considerando 2º

14 VERGARA (2011) pp. 24-25.
} 
su doctora tratante el resultado de unos exámenes que ésta le había solicitado en la última consulta y que arrojaban rangos anormales de prolactina, razón por la que tenía apuro en que ella los revisara. En el momento de mostrarle los resultados -según relata la demandante- la doctora manifiesta que la prolactina estaba sumamente alterada y que podía deberse a un tumor en la hipófisis.

Frente a tal afirmación la demandante entra en pánico y preocupación, por lo que, para confirmar la información, la profesional llama por teléfono al laboratorio, pero nadie contestó porque ya era tarde (cerca de las 20:00 horas). Como no pudo comunicarse con el personal, acudió a los otros médicos que se encontraban trabajando a esa hora para mostrarle los exámenes, y todos concordaron que la alteración del examen se debía a un tumor en la hipófisis.

Añade que salió llorando de la consulta médica y les contó a sus padres lo ocurrido, quienes quedaron con mucha angustia. Además, no pudo repetir el referido examen al día siguiente, puesto que era $1^{\circ}$ de mayo y, por tanto, día feriado.

Afirma, posteriormente, que el 2 de mayo concurrió al centro médico, donde fue informada de un error de digitación en su examen, el que arrojaba 1.353 de prolactina en circunstancias que el nivel era de 13.53, situación que le provocó gran alivio, persistiendo en todo caso en su decisión de repetir el examen cuyos resultados estuvieron listos el 4 de mayo, confirmando que la prolactina estaba normal.

La demandante señala que todo esto le provocó que se ausentara de clases en la carrera de Ingeniería de la Universidad de Valparaíso y perdiera un ramo por no poder rendir el último certamen, debiendo asumir los costos de sicólogo, psiquiatra, universidad y alojamiento.

Finalmente, indica que el Laboratorio Cheul Etcheverry (demandado) incurrió en negligencia al haber entregado un resultado de examen erróneo con niveles prolactina equivocados, lo que constituye un incumplimiento contractual a la luz del artículo 1545 del Código Civil, solicitando se le reparen los daños que ascenderían a la suma total de $\$ 25.000 .000$ (veinticinco millones de pesos).

\section{DEFENSA DEL DEMANDADOY RESOLUCIÓN DE LOS JUECES DE INSTANCIA}

El demandado, Laboratorio Clínico Cheul Etcheverry, solicitó el rechazo de la demanda, indicando que la demandante no alcanzó a estar 48 horas en conocimiento del falso diagnóstico, por lo que existe un expreso ánimo de lucro con la situación producida. Al mismo tiempo, agrega que efectivamente el 30 de abril de 2012 la doctora tratante se percató de algo extraño en los resultados del examen de sangre, explicándole a su paciente la necesidad de repetirlo y al ser consultada sobre las posibles causas, ésta le indicó que podía deberse a un tumor benigno llamado adenoma hipofisario, sin realizar diagnóstico al respecto.

La demandada señala que el examen no constituye diagnóstico médico y que el día 2 de mayo, en no más de 48 horas, se le informó el correcto resultado del examen a la demandante, habiendo una reconfirmación del mismo el día 4 de mayo. Adicionalmente, rechaza la pretensión indemnizatoria respecto de no haber podido rendir la demandante un examen fijado para el día 7 de mayo, de manera que no hubo realmente un impedimento 
para que aquella se presentara, no existiendo relación de causalidad entre el error del resultado de examen de sangre y el daño alegado.

Finalmente, el Tercer Juzgado Civil de Viña del Mar rechazó la demanda en todas sus partes con expresa condenación en costas. Apelado el fallo, la Corte de Apelaciones de Valparaíso revocó la resolución de primer grado, dando por configurado el incumplimiento contractual del Laboratorio demandado, y accediendo a la reparación del daño moral por la suma de $\$ 4.000 .000$ (cuatro millones de pesos) más reajustes e incrementos indicados en la sentencia.

En contra de esta última resolución el demandado dedujo recurso de casación en el fondo, alegando la infracción de los artículos 425 del Código de Procedimiento Civil y 1556 del Código Civil.

\section{LA SENTENCIA DE LA CORTE SUPREMA ROL 1027-2018, DEL 24 DE ENERO DE 2019}

Interpuesto el recurso de casación en el fondo por la parte demandada, la Corte examina las posibles infracciones de ley en que pudieron haber incurrido los jueces de segundo grado. Se indican como infringidos el 425 del Código de Procedimiento Civil y el artículo 1556 del Código Civil. Respecto del primero, se señaló por el recurrente que los jueces dieron por establecido el daño moral por medio de un informe pericial que, a su juicio, no habría sido valorado conforme a las reglas de la experiencia y la lógica. No obstante, el máximo tribunal desecha dicha pretensión porque no hay vulneración de las reglas reguladoras de la prueba, sino únicamente una diferencia en la apreciación de la misma, lo que no puede constituir una infracción de ley a la luz del objeto del recurso de casación en el fondo.

En cuanto a la vulneración del artículo 1556 del Código Civil, la Corte razona sobre la base dos cuestiones que nos parecen relevantes. La primera, relativa a la indemnización propiamente tal del daño moral contractual, la segunda, a la relación causal e imputación objetiva de los daños consecutivos al incumplimiento del demandado.

Respecto del daño moral la Corte Suprema indica, en el considerando sexto de su sentencia, lo siguiente:

"Si bien resulta muy probable que el incumplimiento contractual, cualquiera que sea la naturaleza del contrato, genere sentimientos o sensaciones desagradables, se acepta que no cualquier molestia causada por el incumplimiento contractual debe calificarse jurídicamente como un daño moral" 15 .

En cuanto a la causalidad determinó lo siguiente en el considerando 7o:

"Este par de consideraciones inducen a tener presente aspectos relacionados con la imputación normativa del daño y preguntarse si el daño alegado no se hubiera producido igualmente bajo un comportamiento lícito alternativo; es decir, si la reprobación de uno de los ramos más relevantes de la carrera y, muy probablemente, también de los más complejos no hubiese tenido lugar aun si el laboratorio hubiera informado correctamente el resultado del examen médico. Lo anterior pues, como se ha visto, la recurrente, de una parte, tuvo

15 PARRA CON LABORATORIO CLÍNICO CHEUL ETCHEVERRY (2019), considerando 6o. 
un tiempo suficiente para estudiar y, de otra, había reprobado un enorme número de materias anteriormente.

De esta manera, en opinión de estos sentenciadores, no existen razones suficientes para imputar objetivamente el daño moral que puede haber causado la reprobación de determinadas materias y el consecuente alargamiento de la carrera, al error cometido en el resultado del examen de sangre" 16 .

Los considerandos $6^{\circ}$ y $7^{\circ}$ del fallo resultaron decidores respecto de la alegación hecha por el demandado en su recurso. Ambos sirvieron para que la Corte acogiera la impugnación y determinara que los jueces de segunda instancia incurrieron en infracción de ley.

En términos simples, la sentencia fue invalidada porque las molestias sufridas por la demandante no califican como daño moral indemnizable y, porque dicho daño, además, no es objetivamente imputable al incumplimiento del contrato.

\section{COMENTARIO}

\section{INCUMPLIMIENTO, CAUSALIDAD Y PREVISIBILIDAD}

Hablar sobre causalidad en materia contractual implica tener en consideración dos conceptos relevantes, el primero se refiere a la previsibilidad, mientras que el segundo a la causalidad propiamente tal.

Como ya se ha afirmado más arriba, los estudios dogmáticos sobre la previsibilidad son escasos. Los trabajos existentes no han elaborado, a nuestro juicio, un correcto pronunciamiento sobre la estrecha y profunda relación que tiene la previsibilidad con la causalidad.

En un texto del profesor Hernán Corral se expone, con bastante elocuencia, la relación y diferencia que existe en estos dos conceptos normativos. Se señala que la previsibilidad de que da cuenta el artículo 1558 CC, como norma limitadora de los daños, debe ser considerada al momento de la celebración del contrato, mientras que la previsibilidad que puede estar aparejada al juicio de causalidad normativa (como causa adecuada), ha de considerarse a partir del incumplimiento.

El autor lo explica con las siguientes palabras:

"Para la causalidad de la responsabilidad ese momento es el del hecho ilícito que causa el daño, es decir, tratándose de responsabilidad contractual, el momento del incumplimiento. En cambio, la regla legal de la limitación establece claramente que el momento en que debe valorarse la previsibilidad no es el de la violación del contrato, sino aquel en que el contrato se celebra. En ese sentido, debe entenderse la expresión "al tiempo del contrato" que utiliza el art. 1558 CC"17.

La distinción no es fútil y, desde luego, tiene importantes consideraciones teóricas. Una de ellas es que el juicio de previsibilidad del tiempo del contrato se articula de una forma distinta a la previsibilidad de la causalidad. La primera considera fundamental un aspecto subjetivo del deudor para atribuir los daños que, presunta o realmente, fueron con-

\footnotetext{
16 PARRa CON LABORATORIO CLÍNiCo CHEUl ETCHEVERRY (2019), considerando 7o.

17 Corral (2010) p. 177.
} 
templados al momento de la celebración del negocio. La segunda, imputa objetivamente dichos daños al deudor por su incumplimiento.

Por su parte, el profesor Morales Moreno señala una idea similar en los siguientes términos:

"Una cosa es determinar el bien jurídico protegido, lo cual es función del art. 1107 I, [norma equivalente a nuestro artículo 1558 inciso $1^{\circ} \mathrm{CC}$ ] y otra determinar si un daño producido a un bien jurídico protegido es o no imputable objetivamente al sujeto que materialmente lo ha causado, lo cual es función de las reglas de la imputación objetiva, entre las que se incluye con mayor o menor extensión, la correspondiente a la teoría de la adecuación" 18 .

En otras palabras, ambos autores coinciden -aunque no de forma expresa- en que la previsibilidad del artículo 1558 del Código Civil, en su primer sentido (al tiempo del contrato) es distinta a aquella considerada como parte del juicio de causalidad. En efecto, la primera determina los intereses y derechos lesionables del acreedor cuyo riesgo ha asumido el deudor en caso de incumplimiento ${ }^{19}$, es decir, fija el ámbito o fin de protección del contrato $^{20}$, por lo que el segundo sentido, cumple solamente la función de imputar objetivamente los daños.

A continuación, se revisará la noción del ámbito o fin de protección del contrato y la opinión que tiene la Corte Suprema a su respecto.

\section{2. $\quad$ EL ÁMBITO O FIN DE PROTECCIÓN DEL CONTRATO NO FUE DETERMINANTE}

Como dijimos anteriormente, la previsibilidad de que da cuenta el artículo 1558 del Código Civil, en tanto regla de contemplación de daños, es más bien una regla delimitadora que opera al abrigo del ámbito o fin de protección del contrato, similar a lo que ocurre en materia extracontractual con el fin de protección de la norma ${ }^{21}$.

Esta analogía permite articular un razonamiento similar al que se produce en materia extracontractual, puesto que, en esta sede, el fin de protección de la norma determina los daños de los cuales debe responder el demandado. Y serán aquellos que dicha norma buscaba, precisamente, prevenir o evitar. Por su parte, en la relación contractual, la norma sería equivalente al contrato, y los daños de los que debe responder el deudor son de aquellos que están cubiertos por dicha convención, es decir, se encuentran dentro de su ámbito de resguardo o fin de protección ${ }^{22}$.

18 MORALES (2010) p. 158.

19 SAN MARTÍN (2014) p. 652.

20 De la MAZA (2018b) p. 638. El profesor De la Maza indica "La previsibilidad ex artículo 1558 CC determina el fin de protección del contrato, la previsibilidad propia de la teoría de la causa adecuada sirve, en cambio, para imputar el daño".

21 "La imputación del daño al hecho culpable según el fin protector de la norma se basa en la idea de que los deberes contractuales o legales tienen fines específicos: intentan proteger a ciertas personas, sirven determinados intereses, o pretenden evitar ciertos riesgos. En consecuencia, sólo los daños que se relacionan con los fines específicos que persigue la norma pueden ser imputados a la persona que los ha infringido". Véase BARROS (2006) p. 386.

22 En palabras de Cárdenas y Reveco "El fin de protección del contrato corresponde a lo que en materia extracontractual se ha desarrollado como fin de protección de la norma, criterio fundamental en sistemas típicos de 
La diferencia está, entonces, en que el fin de protección de la norma es un criterio de imputación objetiva, en cambio el fin de protección del contrato es una regla delimitadora objetiva que determina los riesgos que el deudor ha asumido en caso de incumplimiento ${ }^{23}$.

El profesor Morales Moreno ha definido, traduciendo las palabras de Rabel, el concepto de fin de protección del contrato, señalando que: "El fin de protección del contrato determina el interés protegido, lesionable por el incumplimiento del contrato, y fundamentador del derecho a exigir indemnización. Su función no es depurar jurídicamente los cursos de causación del daño contractual, utilizando algunos criterios de causación jurídica, sino definir el interés cuya lesión puede constituir un daño"24.

En ese sentido, el ámbito o fin de protección del contrato si bien se relaciona con el ámbito o fin de protección de la norma de la responsabilidad extracontractual, no son estrictamente lo mismo. Inicialmente se tendería a pensar que operan de una forma equivalente, pero ello -discutiblemente- no es así.

De la misma definición del profesor Morales Moreno se extrae que la función del fin de protección del contrato no es la de ser utilizada como criterio normativo de imputación de daños, sino como mecanismo para determinar los intereses lesionables del acreedor. De manera que toca a otros criterios, distintos del ámbito o fin de protección del contrato, determinar los cursos de causación de daños desde un aspecto jurídico o normativo, pero a partir del incumplimiento.

Así, puede que, de acuerdo al fin de protección del contrato, una determinada convención proteja intereses patrimoniales, extrapatrimoniales o ambos, en caso de incumplimiento del deudor. Para ello, la previsibilidad es una de las herramientas que permiten determinar ese interés cautelado ${ }^{25}$, aunque, naturalmente, no es la única ${ }^{26}$, ya que las

responsabilidad civil; o en hipótesis de culpa infraccional o contra la legalidad en sistemas de cláusulas generales de responsabilidad, como la contenida en el artículo 2314 CC Ch”. Véase CÁRDENAS y REVECO (2018) p. 409.

23 SAN MARTín (2014) p. 652.

24 Morales (2014) p. 115.

25 De la MaZa (2018b) p. 637. En el mismo sentido, la Corte Suprema ha fallado que el ámbito de resguardo o protección del contrato, determina los intereses lesionables del acreedor. Así, en CONSTRUCTORA MONTEVERDE LIMITADA CON MUNICIPALIDAD DE LA LIGUA (2013), considerando 1o "Que, si bien se admite la indemnización del daño moral en la esfera de la responsabilidad contractual, tal como lo ha reconocido esta Corte, concediéndola, ello sólo ha sido aceptado en aquellos casos en que las obligaciones a que da lugar el negocio contractual de que se trata, no se limitan exclusivamente al logro de resultados puramente materiales o patrimoniales.

En efecto, esta clase de resarcimiento procede únicamente en eventos en que la convención extiende su ámbito de resguardo o protección de bienes extrapatrimoniales, o cuando comprende intereses que claramente ya no conciernen al patrimonio sino a la personalidad moral del sujeto, como su afectividad, su estabilidad emocional, su integridad moral, etc".

26 LÓPEZ (2017) p. 317. La autora señala que la naturaleza de la obligación, las circunstancias que rodean la celebración del contrato, entre otros, sirven para fijar el fin de protección del contrato. Así, indica que "La pregunta que debería haber intentado responder la Corte es si el daño moral alegado por la demandante se encontraba protegido por el contrato, esto es, si fue asumido el riesgo de su ocurrencia por el prestador de servicios. Y para responderla habría sido de utilidad que recurriera a criterios que van más allá del contenido extrapatrimonial de la prestación, formulados en el último tiempo por la dogmática nacional y comparada. Tales son, por un lado, la naturaleza de la obligación o de las circunstancias que rodean a esta última, la profesión u oficio del deudor y la información que se le hubiere proporcionado y, por otro, la función típica del contrato y el propósito práctico perseguido por los contratantes". 
circunstancias y características particulares de la referida convención también sirven para determinar el interés comprometido del acreedor ${ }^{27}$.

Ahora bien, para la determinación del daño indemnizable por incumplimiento del laboratorio demandado, la misma sentencia excluye el ámbito o fin de protección del contrato como mecanismo de delimitación. Esto lo hace en el considerando $6^{\circ}$ cuando indica que "[e]l tipo de contrato que se está examinando aquí, uno relativo a prestaciones de carácter médico, sin duda, protege intereses extrapatrimoniales; las personas, por lo general no se hacen exámenes con el objeto de aumentar su patrimonio, sino de velar por su salud. Por lo mismo, esta primera limitación al daño moral en materia contractual, correspondiente a la determinación del tipo de intereses que protege el contrato, no ha de aplicarse". ${ }^{28}$ (énfasis añadido).

Resulta a lo menos curioso por qué no ha de aplicarse el ámbito o fin de protección del contrato, especialmente si, en el caso sub lite, se trata de un contrato médico. Pero la verdad es que la respuesta, aunque no tan obvia, sí es extraordinariamente persuasiva y, según entiendo, consiste en que las afectaciones reclamadas por la demandante a título de daño moral deben ser acomodadas no solamente con el primer examen de delimitación (fin de protección), sino también por medio de criterios normativos de causalidad, dada las consecuencias remotas a las que se hicieron referencia y cuya fuente se imputa al incumplimiento del deudor.

En otras palabras, tal como ocurre en materia extracontractual con el fin de protección de la norma, el fin de protección del contrato cumpliría únicamente la función de determinar el interés directamente cautelado por la convención. Por ende, cuando se intenta imputar objetivamente daños consecutivos, o que en algún sentido escapan a los que el contrato cautela, deberán utilizarse criterios distintos del ámbito de protección para establecer la extensión del daño indemnizable ${ }^{29}$, como por ejemplo, el criterio de adecuación, aumento del riesgo, etc. De manera que hace bien la Corte cuando considera los otros dos factores que a continuación se desarrollan.

27 BAZAN CON SOCIEDAD COMERCIAL CONCESIONARIA SUBTERRA S.A. (2012), considerando 10º que señala: "Que estima este tribunal de casación que para analizar la procedencia del daño moral por la infracción de un contrato, no es suficiente, para descartarlo, la mera constatación de que la convención, por su naturaleza, no protege intereses extrapatrimoniales o comprende intereses que claramente pueden afectar a la personalidad del sujeto, sino que es menester detenerse en las particulares circunstancias y características en que se celebró el que vincula a las partes, pues de ellas fluirá la posibilidad de que la mencionada infracción provoque un daño extrapatrimonial".

28 PARRA CON LABORATORIO CLÍNICO CHEUL ETCHEVERRY (2019), considerando 6º.

29 BARRos (2006) p. 390. "La idea del fin protector de la norma usualmente sólo permite limitar la responsabilidad por el primer daño que sufre la víctima. Ello es una consecuencia de la relación que debe haber, según este criterio de atribución, entre la culpa y el daño. Pero ocurre que la culpa se agota en la valoración del primer daño y no se transmite a las preguntas relativas a la imputación de los daños consecuentes". Luego el autor afirma: "Se comprueba, entonces, que el fin de la norma no es un criterio para determinar la proximidad que deben tener los daños subsecuentes para que queden cubiertos por la responsabilidad de quien provocó ese primer daño. Esa pregunta se refiere a los criterios para calificar esos daños consecuentes como directos o indirectos (...)”. BARROS (2006) p. 391. 


\section{LOS CONTORNOS DEL DAÑO MORAL Y LA ENTIDAD DE LA AFECTACIÓN COMO FACTOR EXCLUYENTE}

El segundo criterio de delimitación empleado por la Corte Suprema para excluir la indemnización del daño moral alegado por la demandante se refiere a la entidad del daño sufrido. Aquí la Corte nos invita a reflexionar sobre un mecanismo delimitador que se funda en el concepto normativo de daño moral con las siguientes palabras: "[a]sí las cosas, la segunda limitación que experimenta la indemnización por daño moral en materia contractual se relaciona con la entidad del sufrimiento, malestar, incomodidad, angustia u otro sentimiento o sensación desagradable que se alega”.

La dogmática civil tradicional coincide plenamente en que uno de los requisitos para que el daño sea indemnizable es que debe tener cierta entidad ${ }^{30}$. En consecuencia, se excluyen las molestias e incomodidades que puede haber provocado el incumplimiento contractual, ya que, en palabras de la profesora Carmen Domínguez, "[l]a infracción del contrato supone siempre molestias o desagrados, pero ello no significa que éstos puedan y deban ser indemnizados como daño moral" 31 .

Esto implica que toda relación contractual lleva envuelta un riesgo de sufrir un pesar o molestia ante su infracción. De manera que, para que dichas afectaciones sean efectivamente indemnizables, deben haber superado un cierto umbral de tolerancia o lo que razonablemente puede soportar cualquier persona en las mismas circunstancias. Se trata, en consecuencia, de establecer que ciertas afectaciones son propias de las relaciones contractuales e, incluso, de la vida en sociedad ${ }^{32}$.

Por otra parte, aunque la magnitud o gravedad del daño sea un factor relevante para considerarlo o no como indemnizable, la Corte agrega, a nuestro parecer, otro que no tiene que ver con la intensidad, sino más bien con la duración del mismo.

Ambos factores, intensidad y duración, sirvieron para calificar la afectación alegada por la demandante como un daño no indemnizable. Respecto de la duración, el máximo tribunal hizo énfasis en el poco tiempo que la demandante estuvo expuesta a la situación que le causo angustia, no más de 48 horas, indicando lo siguiente al respecto:

30 Por todos, BARros (2006) p. 226. "La noción de daño excluye aquellas incomodidades o molestias que las personas se causan recíprocamente como consecuencia normal de la vida en común”.

31 Domínguez (2000) p. 355. En el mismo sentido se resolvió en CIFUENTES CON AUTOMOTRIZ ROSSELOT S.A. (2015), que en el voto de minoría se manifiesta "Que tampoco existe en autos prueba alguna de que lo que se reclama a título de daño moral sea una afectación distinta a la simple molestia o perturbación propia de todo incumplimiento contractual. En tal sentido, conviene tener presente que la sola infracción de un contrato no da lugar a una reparación por daño moral porque la sola molestia, perturbación o desagrado que le genera al acreedor la infracción no es suficiente para configurarle como tal. De lo contrario, se llegaría al exceso de que todo incumplimiento contractual generaría un daño moral derivado de contrato dado que esas consecuencias son propias a toda infracción”. También SILVA CON CENTRAL PARKING SYSTEM CHILE S.A. (2015), considerando 3º. 32 GONZÁLEZ (2017) p. 200. Los tribunales también han reaccionado en la misma dirección, por ejemplo, NO SE CONSIGNA CON NO SE CONSIGNA (2006), considerando $4^{\circ}$ : "Que, en lo que concierne al daño moral reclamado por el actor, si bien es posible su reparación incluso en la responsabilidad contractual, como ya es doctrina constante, no es posible confundirlo con los desagrados, disgustos o preocupaciones que origina, naturalmente todo incumplimiento de un contrato pues entonces, la existencia del daño moral sería una consecuencia casi necesaria de toda infracción contractual”. En el mismo sentido, JARAMILLO CON HERNANDEZ MOTORES S.A. (2014), considerando $5^{\circ}$. 
"De esta manera, si lo que se reclama como daño moral es el sufrimiento que padeció la recurrente durante ese breve lapso (de tiempo), estos sentenciadores estiman que, no obstante resultar plausible que alguien pueda sufrir por la incertidumbre que genera el elenco de posibilidades que puede explicar un indicador inusualmente alto, dicho padecimiento, particularmente si se mantiene por ese lapso tan breve, no resulta suficiente para configurar un daño moral"33 (énfasis añadido).

La duración del daño moral alegado, por sí solo considerado, aunque sea breve, en principio no debería ser motivo para excluir la reparación pretendida por la víctima. Puede ocurrir, por ejemplo, que una persona sufra una grave aflicción por un espacio breve de tiempo, y no por ello se debe desechar la pretensión indemnizatoria. Lo mismo debería pensarse en casos -como los que tienen lugar en derecho del consumo- en que una persona sufre una intoxicación estomacal por la ingesta de alimentos en mal estado, y que no se extiende por más de 24 horas. La falta de seguridad y la vulneración de la salud, aunque sea por poco tiempo, legitimaría sin duda alguna una acción indemnizatoria por daño moral.

Ahora bien, lo que hace la Corte es complementar el criterio o factor temporal del daño alegado, con la importancia o gravedad del mismo. En el caso, dada la forma en cómo se desarrollaron los acontecimientos, las meras molestias y preocupaciones fueron las que, sumada a la temporalidad, llevaron al tribunal a desestimar la reparación solicitada.

En síntesis, aunque se trata de criterios cualitativamente distintos, se observa una clara inclinación de la Corte en estimar la importancia global de la afectación alegada por la demandante. La duración y la gravedad mínimas, no califican, a su juicio, como daño moral indemnizable.

\section{EL CRITERIO DE INCREMENTO DEL RIESGO UTILIZADO POR LA CORTE SUPREMA PARA EXCLUIR LOS DAÑOS CONSECUTIVOS}

Hemos hecho una larga referencia a la previsibilidad como regla limitadora de los daños contractuales, en cuanto ayuda a determinar el ámbito o fin de protección del contrato, y también como un criterio de imputación objetiva, como causa adecuada.

En principio, las cosas pudieron haberse resuelto aplicando el criterio de adecuación para imputar objetivamente los daños consecutivos al incumplimiento del demandado, en tanto ellos no forman parte de la cadena causal que se esperaría de acuerdo al curso ordinario de los acontecimientos ${ }^{34}$.

Sin embargo, nos parece que la Corte utilizó un criterio de imputación distinto, para ello deberá tenerse en consideración el daño consecutivo alegado y el texto de la sentencia.

Respecto del daño consecutivo, cabe hacer presente que la demandante alegó que el error en el resultado del examen por el Laboratorio, además de la preocupación, provocó que haya reprobado una asignatura de la carrera de Ingeniería que cursaba en la Universidad de Valparaíso, solicitando que esta situación sea compensada a título de daño patrimonial y moral.

33 PARRA CON LABORATORIO CLÍNICO CHEUL ETCHEVERRY (2019), considerando 6º párrafo final.

34 Barros (2006) p. 396 
Por su parte, la Corte manifiesta un profundo desacuerdo con lo pretendido por la actora, puesto que, en el considerando $7^{\circ}$, indica lo siguiente:

"Este par de consideraciones inducen a tener presente aspectos relacionados con la imputación normativa del daño y preguntarse si el daño alegado no se hubiera producido igualmente bajo un comportamiento lícito alternativo; es decir, si la reprobación de uno de los ramos más relevantes de la carrera y, muy probablemente, también de los más complejos no hubiese tenido lugar aun si el laboratorio hubiera informado correctamente el resultado del examen médico. Lo anterior pues, como se ha visto, la recurrente, de una parte, tuvo un tiempo suficiente para estudiar y, de otra, había reprobado un enorme número de materias anteriormente.

De esta manera, en opinión de estos sentenciadores, no existen razones suficientes para imputar objetivamente el daño moral que puede haber causado la reprobación de determinadas materias y el consecuente alargamiento de la carrera, al error cometido en el resultado del examen de sangre" 35 .

De estas consideraciones se puede extraer que la Corte Suprema ha aplicado un criterio de imputación diverso al de la causa adecuada para excluir la reparación de los daños consecutivos. Lo que ha utilizado, a nuestro parecer, es el criterio de incremento del riesgo o del riesgo incremental. Esto significa que deben excluirse los daños que, aun si el demandado hubiese adoptado una conducta lícita, tienen una probabilidad semejante de haberse producidos $^{36}$.

En este sentido, el profesor Enrique Barros nos explica lo siguiente sobre este criterio de imputación: "[e]l aumento del riesgo resulta de comparar los efectos de la conducta culpable con la conducta diligente alternativa; si el daño hubiese tenido una probabilidad semejante de ocurrir en el evento que el demandado se hubiese comportado diligentemente, puede asumirse que no es atribuible al hecho del demandado, porque no incrementó el riesgo de su materialización" 37 .

En efecto, analizando lo expresado por la Corte en el ya referido considerando séptimo, especialmente cuando señala que: "Este par de consideraciones inducen a tener presente aspectos relacionados con la imputación normativa del daño y preguntarse si el daño alegado no se hubiera producido igualmente bajo un comportamiento lícito alternativo (...)", está claramente revisando la extensión de los daños consecutivos alegados, consistente en la reprobación de la asignatura, bajo el modelo del criterio del aumento del riesgo.

Esos daños consecutivos, en tanto generaron un detrimento patrimonial y moral en la demandante, resultaron no ser objetivamente imputables a la conducta (incumplimiento) del demandado. Y esto, porque, aun en el evento de haberse informado correctamente

35 PARRA CON LABORATORIO CLÍNICO CHEUL ETCHEVERRY (2019), considerando 7o, párrafos 4 a 6.

36 Aquí es donde la Corte evidencia este criterio de imputación objetiva "Este par de consideraciones inducen a tener presente aspectos relacionados con la imputación normativa del daño y preguntarse si el daño alegado no se hubiera producido igualmente bajo un comportamiento lícito alternativo; es decir, si la reprobación de uno de los ramos más relevantes de la carrera y, muy probablemente, también de los más complejos no hubiese tenido lugar aun si el laboratorio hubiera informado correctamente el resultado del examen médico". PARRA CON LABORATORIO CLÍNICO CHEUL ETCHEVERRY (2019), considerando 7o .

37 BARROS (2006) p. 402. 
el resultado del examen médico por el laboratorio, nada asegura que la actora hubiese aprobado la materia en cuestión. En otras palabras, si suprimimos la conducta que se le reprocha al Laboratorio, no hay certeza de que la demandante hubiese tenido éxito en su examen de asignatura, de forma tal que el daño alegado por ésta (consistente en la reprobación del ramo), no es imputable normativamente a aquel, al no haber aumentado el riesgo de su ocurrencia ${ }^{38}$.

En consecuencia, hace bien la Corte Suprema al rechazar la pretensión indemnizatoria de la actora, puesto que, si bien la reprobación de una asignatura puede significar un importante menoscabo patrimonial y/o una afectación moral que sobrepasa la simple molestia, no existe finalmente conexión o relación de causalidad entre dicha afectación y el incumplimiento que se le imputa al demandado, de acuerdo al criterio normativo empleado.

\section{CONCLUSIONES}

1. La regla de la previsibilidad del artículo 1558 del Código Civil ha sido poco estudiada por la dogmática civil, y poco trabajada por la jurisprudencia, en cuanto herramienta de delimitación de daños contractuales. Esto ha llevado a que se haya estructurado como regla de contemplación de daños con carácter subjetivo, que de a poco ha comenzado a objetivarse bajo el modelo de asunción de riesgos por el deudor.

2. De acuerdo a la doctrina nacional y extranjera la misma regla del artículo 1558 del Código Civil ha servido para considerar el elemento previsibilidad con un doble enfoque. El primero como mecanismo para determinar los intereses protegidos por el contrato y los riesgos asumidos por el deudor, mientras que el segundo está incorporado en el juicio de causalidad como causa adecuada.

3. El problema que se suscitó en la presente sentencia consistió en determinar dos cosas: a) el concepto de daño moral y; b) si los daños consecutivos (patrimonial y moral) pueden ser atribuidos al incumplimiento del demandado.

4. Como la previsibilidad del art. 1558 del Código Civil, en tanto regla de contemplación, sirve para determinar los intereses y riesgos del contrato, esto es, el fin de protección del mismo, la Corte Suprema, no obstante, consideró que este no era el mecanismo idóneo de delimitación para llegar a la solución. El fin de protección del contrato fue descartado.

5. De cara al procedimiento de delimitación, la Corte se basa en dos cuestiones relevantes. La primera en el concepto normativo de daño moral, la segunda, al criterio de imputación objetiva para establecer la relación de causalidad.

6. La noción de daño moral obliga a excluir las molestias por incumplimiento contractual. Esto porque los requisitos generales de la responsabilidad civil exigen siempre una

\footnotetext{
38 "En esencia, los daños consecuentes no pueden ser objetivamente imputados al hecho inicial cuando se trata de simples riesgos generales de la vida, que sólo casual o circunstancialmente están relacionados con el hecho ilícito". BARROS (2006) p. 402. En un sentido similar, CÁRDENAS y REVECO (2018) p. 399, señalan que "Por otro lado, el incremento de los riesgos generales de la vida permite excluir como causante de los daños sufridos por el acreedor a aquellos incumplimientos contractuales que no incrementan el riesgo general que soportan las partes en el normal tráfico comercial en que se desenvuelven".
} 
entidad mínima para que un daño sea resarcible, de lo contrario, este siempre debería ser indemnizado, porque todo incumplimiento produce molestia o disgusto.

7. Sobre la causalidad, el criterio de imputación objetiva que fue utilizado para excluir la indemnización de los daños consecutivos (reprobación de asignatura) fue el incremento del riesgo. Esto, porque la materialización del daño era también probable aun con ocasión de un comportamiento lícito del demandado. Es decir, su conducta no aumentó el riesgo de ocurrencia. Por lo tanto, las afectaciones alegadas por la demandante pertenecen más bien al ámbito de riesgos generales de la vida y no a una conducta imputable al Laboratorio.

\section{BIBLIOGRAFÍA CITADA}

ALESSANDRI RODRÍGUEZ, Arturo (1943): De la responsabilidad extracontractual en el Derecho Civil Chileno (Santiago, Imprenta Universitaria)

BARRIENTOS, Marcelo (2007): "Los daños extrapatrimoniales que se previeron o pudieron preverse al tiempo del contrato", Revista Chilena de Derecho, Vol. 34, No 1, pp. 7-22.

BARROS BOURIE, Enrique (2006): Tratado de responsabilidad extracontractual (Santiago, Editorial Jurídica de Chile)

Barros Errázuriz, Alfredo (1932): Curso de Derecho Civil. Segundo Año (Santiago, Editorial Nascimiento).

BURROWS, Andrew (2005): Remedies for Torts and breach of contract (Oxford, Oxford University Press, 3rd ed.)

BUTRÓN FIRPO, Roberto (2009): "La indemnización del daño moral en nuestra legislación” en Tavolari, Raúl (Dir.) Doctrinas Esenciales Derecho Civil. Responsabilidad Extracontractual (Santiago, Editorial Puntolex).

Cárdenas Villarreal, Hugo y Reveco UrzÚa, Ricardo (2018): Remedios Contractuales. Cláusulas, acciones y otros mecanismos de tutela del crédito (Santiago, Thomson Reuters).

CORRAL TALCiAni, Hernán (2009): "La regla de la previsibilidad de los daños contractuales: un ejemplo de la influencia de la doctrina de Pothier en las tradiciones de Derecho civil continental y de Common law", Revista de Derecho Universidad Católica del Norte, Año 16, No 2, pp. 139-159.

Corral TalCiani, Hernán (2010) Contratos y daños por incumplimiento (Santiago, AbeledoPerrot)

DE lA MAZA, Íñigo (2018a): "El daño moral en materia contractual: la mirada de la Corte Suprema", Revista Chilena de Derecho, Vol. 45 No 2, pp. 275-309.

DE la MAZA, Íñigo (2018b): "Previsibilidad y causalidad", Estudios de Derecho Civil XIII, (Santiago, Thomson Reuters)

FUeYo LANERI, Fernando (1965): "Sobre el daño extrapatrimonial en el incumplimiento de contrato" Revista Jurídica de Buenos Aires.

FUeYo LANERI, Fernando (1972): "De nuevo sobre el daño extrapatrimonial en el cumplimiento de contrato" Colección Justitia et Jus, Sección de Investigaciones, No 8.

GonZÁlez CAZOrla, Fabián (2017): "Delimitación del daño moral a través de consideraciones de justicia distributiva”, Revista de Derecho de la Universidad de Concepción, No 242, pp. 191-220. 
GonZÁleZ CAZORla, Fabián (2019): Daño moral en el Derecho del Consumidor (Santiago, DER Ediciones).

LÓPEZ DíAZ, Patricia (2017): “Indemnización de daños autónoma por incumplimiento contractual. Vencimiento del término esencial. Infracción de obligaciones de advertencia. Daño moral y esfera de protección del contrato. Corte Suprema, 14 de noviembre de 2016, rol 5746-2016. Cita en línea Legal Publishing CL/JUR/7557/2016", Revista Chilena de Derecho Privado, sección comentarios de jurisprudencia, No 28, pp. 307-321.

Morales Moreno, Antonio Manuel (2010): Incumplimiento del contrato y lucro cesante (Navarra, Thomson Reuters)

Morales Moreno, Antonio Manuel, (2014): "Claves de la modernización del derecho de contratos”, en De la Maza et al., Estudios de derecho de contratos. Formación, cumplimiento e incumplimiento (Santiago, Thomson Reuters).

Pizarro Wilson, Carlos (2013): "Contra el fatalismo del perjuicio. A propósito del deber de mitigar el daño", Revista de Derecho de la Pontificia Universidad Católica de Valparaíso, XLI, pp. 69-82.

SAN MARTín NeIRA, Lilian (2014): "La previsibilidad como límite al resarcimiento del daño por incumplimiento contractual”, en Susan Turner y Juan Andrés Varas (coords.) Estudios de Derecho Civil IX (Santiago, Thomson Reuters).

Vergara Benzanilla, José Pablo (2011): "Comentarios sobre el daño moral en materia contractual”, Revista de Derecho del Consejo de Defensa del Estado, No 26, pp. 11-26.

\section{JURISPRUDENCIA CITADA}

BAZAN CARDEMIL, JORGE CON SOCIEDAD COMERCIAL CONCESIONARIA SUBTERRA S.A. (2012): Corte Suprema, 1 de octubre de 2012, Rol 4415-2010, Thomson Reuters CL/ JUR/2153/2012.

Cifuentes, ANDRÉs IGNACIO CON Automotriz Rosselot S.A., (2015): Corte de Apelaciones de Santiago, 23 de enero de 2015, Rol 1552-2014, Thomson Reuters CL/ JUR/375/2015.

CONSTRUCTORA MONTEVERDE LIMITADA CON MUNICIPALIDAD DE LA LIGUA (2013): Corte Suprema, 26 de septiembre de 2013, Rol 375-2013, Thomson Reuters CL/ JUR/2114/2013.

JARAMILLO INOSTROZA, SANDRA CON HERNANDEZ MOTORES S.A., (2014): Corte de Apelaciones de Santiago, 17 de noviembre de 2014, Rol 1110-2014, considerando 5º, Thomson Reuters CL/JUR/8721/2014.

NO SE CONSIGNA CON NO SE CONSIGNA (2006): Corte de Apelaciones de Concepción, 10 de agosto de 2006, Rol 8190-2003, Thomson Reuters CL/JUR/2700/2006.

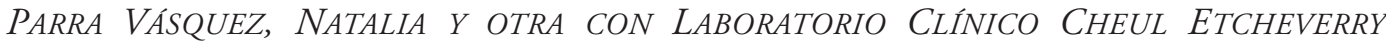
(2019): Corte Suprema, 24 de enero de 2019, Rol 1027-2018, Thomson Reuters CL/ JUR/447/2019.

RAFART, MARÍA CON BANCO DE CHILE (1994): Corte Suprema, Rol 18647-1994, de fecha 20 de octubre de 1994 Thomson Reuters CL/JUR/1624/1994. 
GONZÁLEZ CAZORLA, Fabián — "Imputación objetiva de los daños por incumplimiento contractual"

Ruiz, Hugo con laboratorio Biológico S.A. y otros (2001): Corte Suprema, 5 de noviembre de 2001, Rol 1368-2000, Identificador Thomson Reuters CL/ JUR/4636/2001.

Silva, Juan Matías con Central PARKing System Chile S.A., (2015): Corte de Apelaciones de Santiago, 19 de febrero de 2015, Rol 1691-2014, Thomson Reuters CL/ JUR/1040/2015.

Zorin S.A. CON COMPAÑÍ SidERÚRGiCA HUACHIPATO (2012): Corte Suprema, 31 de octubre de 2012, Rol 3325-2012, Thomson Reuters Cl/JUR/2412/2012. 